\title{
Deterministic entanglement of two neutral atoms via Rydberg blockade
}

\author{
X. L. Zhang, L. Isenhower, A. T. Gill, T. G. Walker, and M. Saffman \\ Department of Physics, University of Wisconsin, \\ 1150 University Avenue, Madison, WI 53706 USA
}

\begin{abstract}
We demonstrate the first deterministic entanglement of two individually addressed neutral atoms using a Rydberg blockade mediated controlled-NOT gate. Parity oscillation measurements reveal an entanglement fidelity of $F=0.58 \pm 0.04$, which is above the entanglement threshold of $F=0.5$, without any correction for atom loss, and $F=0.71 \pm 0.05$ after correcting for background collisional losses. The fidelity results are shown to be in good agreement with a detailed error model.
\end{abstract}

PACS numbers: 03.67.-a, 32.80.Qk, 32.80.Ee

Entangled states are a crucial resource for quantum information processing (QIP) and quantum communication. Entangled states have been demonstrated with several different physical systems including trapped ions 1, 2], superconductors [3], photons [4] and atomic systems [57]. Recent experiments using Rydberg state mediated interactions of neutral atoms [8] have demonstrated bipartite correlations just below the threshold of $F=0.5$ for quantum entanglement [9, 10]. Correction of the measured data for atom loss in those experiments revealed post-selected or a posteriori entanglement with fidelities of $F=0.58 \pm .07[10]$ and $F=0.75 \pm .07[9]$. While $a$ posteriori entanglement is useful for tests of Bell inequalities and studies of quantum non-locality, states with $F<0.5$ cannot be directly purified to cross the quantum boundary of $F>0.5$.

In this letter we report on Rydberg blockade experiments using improved experimental techniques that demonstrate deterministic preparation of entangled states with $F>0.5$ without any correction for atom loss during the entire state preparation, gate operation, and measurement sequence. Applying a correction for losses due to background collisions that are independent of the gate operation we demonstrate entanglement fidelity of $F=0.71 \pm .05$. These results demonstrate unambiguously that Rydberg blockade can deterministically generate entangled states which are a crucial resource for QIP.

It was first proposed in [8] to use Rydberg blockade interactions to implement a two-qubit entangling gate with neutral atoms. The basic idea behind Rydberg blockade is straightforward: excitation of a control atom to a Rydberg state prevents subsequent excitation of a target atom provided the dipole mediated Rydberg-Rydberg blockade shift B is large compared to the excitation Rabi frequency $\Omega$. If the control atom excitation is state dependent we get a conditional phase shift on the target atom since excitation and de-excitation of the target atom adds a $\pi$ phase shift to the wavefunction. This conditional phase gate $C_{Z}$ is converted into a controlledNOT gate $\left(H-C_{Z} \mathrm{CNOT}\right)$ using single qubit $\pi / 2$ rotations (Hadamard gates) on the target atom before and after the controlled phase operation.
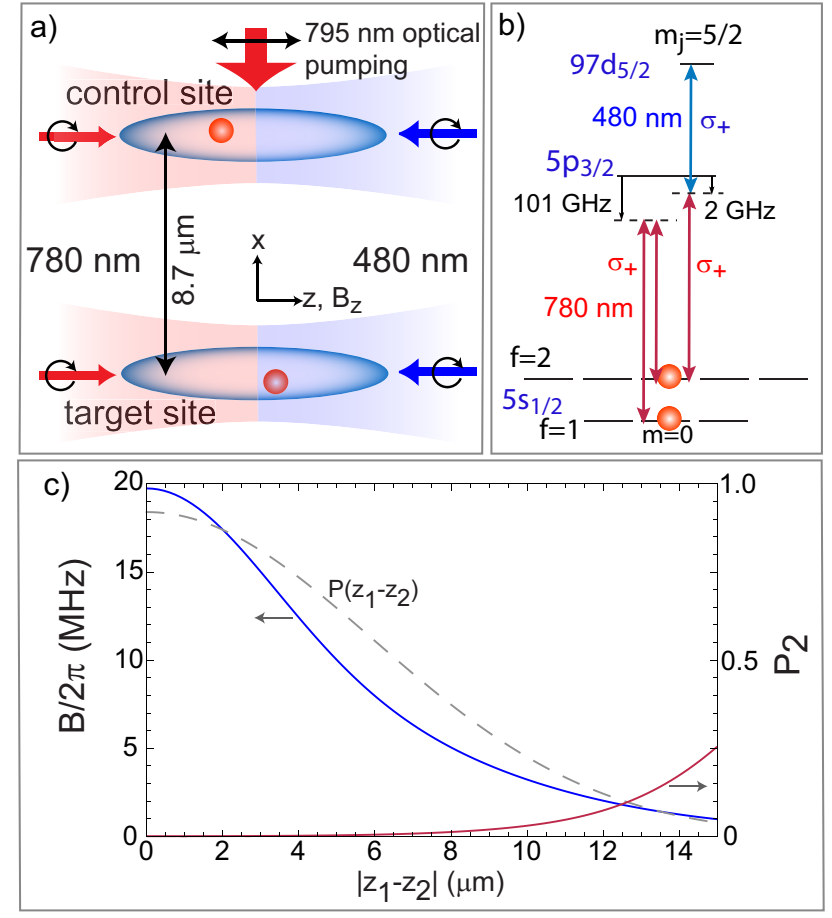

FIG. 1: (color online) a) Experimental geometry, b) relevant levels of ${ }^{87} \mathrm{Rb}$, and c) calculated blockade shift B and double excitation probability $P_{2}$. The relative probability distribution $P\left(\left|z_{1}-z_{2}\right|\right)$ assumes a trapping potential $U / k_{B}=4.5 \mathrm{mK}$, waist of the $1064 \mathrm{~nm}$ trapping light of $w=3.2 \mu \mathrm{m}$ and atom temperature $T=175 \mu \mathrm{K}$.

The intrinsic error of the controlled phase operation can be estimated from[11, 12]

$$
E=\frac{7 \pi}{4 \Omega \tau}\left(1+\frac{\Omega^{2}}{\omega_{10}^{2}}+\frac{\Omega^{2}}{7 \mathrm{~B}^{2}}\right)+\frac{\Omega^{2}}{8 \mathrm{~B}^{2}}\left(1+\frac{6 \mathrm{~B}^{2}}{\omega_{10}^{2}}\right) .
$$

In our experiments $\tau \sim 300 \mu \mathrm{s}$ is the radiative lifetime of the $97 d_{5 / 2}$ Rydberg level, $\omega_{10}=2 \pi \times 6.83 \mathrm{GHz}$ is the splitting of the ${ }^{87} \mathrm{Rb}$ qubit basis states, and $\Omega=$ $2 \pi \times 0.81 \mathrm{MHz}$. In the experimental geometry shown in Fig. 1 a range of two-atom separations, and hence blockade shifts, occur. The blockade shift curve shown in Fig. [1. was calculated from the theory of Ref. [13] using a trap separation of $x=8.7 \mu \mathrm{m}$ and a bias magnetic field 
of $B_{z}=0.37 \mathrm{mT}$ applied along the $\hat{z}$ axis. Averaging Eq. (1) over the probability distribution $P\left(\left|z_{1}-z_{2}\right|\right)$ gives an expected error of $E=6.5 \times 10^{-3}$. The corresponding averaged blockade shift from Eq. (11) is $B=2 \pi \times 5.3 \mathrm{MHz}$. The spatial distribution $P\left(\left|z_{1}-z_{2}\right|\right)$ is dependent on the trapped atom temperature which is estimated to be $175 \mu \mathrm{K}$ based on compatibility of three different diagnostics: release and recapture measurements 14], comparison of the atomic spatial distribution found from averaged camera images to that expected given our trapping beam waist and power [15], and measurement of the radial vibrational frequency from parametric heating data. Uncertainties in the beam waist and trapping power lead to a $\sim \pm 20 \%$ uncertainty in the atom temperature. This uncertainty does not significantly affect the estimated intrinsic gate error.

This calculation shows that the intrinsic error of the Rydberg blockade gate is less than $1 \%$ with available experimental parameters. As we show below the observed gate fidelity errors are a factor of 10 or more higher than our theoretical limit, the reason being that Eq. (1) does not account for all experimental effects and technical imperfections. Of particular importance are Doppler effects which both degrade the Rydberg excitation fidelity and add a stochastic phase which limits the entanglement fidelity. In what follows we present experimental results showing improved CNOT truth table fidelity and deterministic two-atom entanglement. We then identify the dominant error sources leading to loss of fidelity, and suggest ways in which the theoretical error limit could be reached in future work.

The experimental apparatus and procedures have been described in detail in our recent publications [10, 15]. Here we describe the procedures briefly and highlight the changes relative to our previous work. Single atoms with temperatures of $T \sim 250 \mu \mathrm{K}$ are loaded from a magneto-optical trap into optical traps created by focusing $1064 \mathrm{~nm}$ light to waists of $w=3.2 \mu \mathrm{m}$. Using a diffraction grating we create a linear array of 5 trapping sites which can be individually controlled 16]. In the present work we use two of the central sites with a separation of $x=8.7 \mu \mathrm{m}$. This is slightly smaller than in our previous CNOT experiment $(x=10 \mu \mathrm{m})$ which gives about 2.3 times more blockade shift in the van der Waals limit. A bias magnetic field is applied along $z$, which defines the quantization axis for optical pumping (using $B_{z}=0.15 \mathrm{mT}$ ) and lifts the degeneracy of the Rydberg state Zeeman sublevels (using $B_{z}=0.37 \mathrm{mT}$ ) during gate operation.

The experimental sequence is shown in Fig. 3a. After loading into the optical traps an atom number measurement is made to verify the presence of two atoms, followed by a $20 \mathrm{~ms}$ cooling phase (light detuned by $-3 \gamma$ from the cycling transition). The cooling phase gives $T \sim 175 \pm 35 \mu \mathrm{K}$ atoms which are then optically pumped into $\left|f=2, m_{f}=0\right\rangle$ using $\pi$ polarized light propagating along $-x$ tuned to the $\left|5 s_{1 / 2}, f=2\right\rangle \rightarrow\left|5 p_{1 / 2}, f^{\prime}=2\right\rangle$ D1 transition at $795 \mathrm{~nm}$ and $780 \mathrm{~nm}$ light tuned to the $\left|5 s_{1 / 2}, f=1\right\rangle \rightarrow\left|5 p_{3 / 2}, f^{\prime}=2\right\rangle \mathrm{D} 2$ transition. The $5 p_{1 / 2}$ level has a larger excited state hyperfine splitting than $5 p_{3 / 2}$ and we thereby obtain an improved optical pumping efficiency compared to [10] of about 0.99. Readout of the qubit state of the atoms is performed by using light forces to remove one of the hyperfine states [10] and then collecting resonance fluorescence on a cooled EMCCD camera from a region of interest with predetermined thresholds indicating the presence or absence of a single atom. Typical integration times were approximately $10 \mathrm{~ms}$.

For the qubit basis we use the ground hyperfine states $|0\rangle \equiv\left|f=1, m_{f}=0\right\rangle,|1\rangle \equiv\left|f=2, m_{f}=0\right\rangle$. Single qubit rotations $|0\rangle \leftrightarrow|1\rangle$ use two-photon stimulated Raman transitions driven by focusing a $\sigma_{+}$polarized $780 \mathrm{~nm}$ laser with frequency components separated by $\omega_{10}$ and detuned by $\Delta / 2 \pi=-101 \mathrm{GHz}$ to the red of the D2 transition [17]. Typical total power in the two Raman sidebands is $\sim 90 \mu \mathrm{W}$ and we achieve $\pi$ pulse times of $\sim 900$ ns as shown in Fig. 2, with peak-peak amplitude of better than 0.98 after correction for background atom loss of $10 \%$. The single atom loss probability during the gap of $0.11 \mathrm{~s}$ between state preparation and output state measurement has been reduced from $15 \%$ in earlier work to typically $10 \%$ (see Fig. 3a). This was achieved using faster mechanical shutters for laser beams and faster magnetic field switching electronics.

Coherent Rydberg excitation between $|1\rangle$ and $|r\rangle=$ $\left|97 d_{5 / 2}, m_{j}=5 / 2\right\rangle$ uses a two photon transition with $\sigma_{+}$ polarized 780 and $480 \mathrm{~nm}$ beams. The $780 \mathrm{~nm}$ beam is tuned by about $\Delta_{f=2}=-2 \pi \times 2 \mathrm{GHz}$ to the red of the D2 line. Typical beam powers are $2.4 \mu \mathrm{W}(13 \mathrm{~mW})$ with $x-y$ averaged beam waists of $7.7(4.5) \mu \mathrm{m}$ at $780(480) \mathrm{nm}$ giving Rydberg $\pi$ pulse times of $\sim 620$ ns with peak-peak amplitude from a least squares fit of 0.92 after correction for background atom loss of 10\%, as shown in Fig,2b. Improved long term stability of the pulse areas for ground state and Rydberg operations has been established using a system that monitors the powers transmitted through single mode fibers to the experimental chamber $(20 \mathrm{~ms}$ diagnostic slice at the end of each sequence in Fig. $3 \mathrm{a}$ ), and corrects the incident beam powers accordingly [18].

Using the above methods we have generated the CNOT probability truth table shown in Fig. 3. As input to the CNOT sequence we apply the four two-qubit computational states which can be prepared with an average fidelity of 0.97 , as shown in Fig. 3b). The measured CNOT probability truth table shown in Fig. 3r has the values

$$
\left|U_{\mathrm{CNOT}}\right|=\left(\begin{array}{cccc}
0.08 & 0.93 \pm .06 & 0 & 0 \\
0.88 \pm .06 & 0.02 & 0.02 & 0.02 \\
0 & 0 & 0.90 \pm .07 & 0.05 \\
0.02 & 0.05 & 0.07 & 0.94 \pm .06
\end{array}\right)
$$



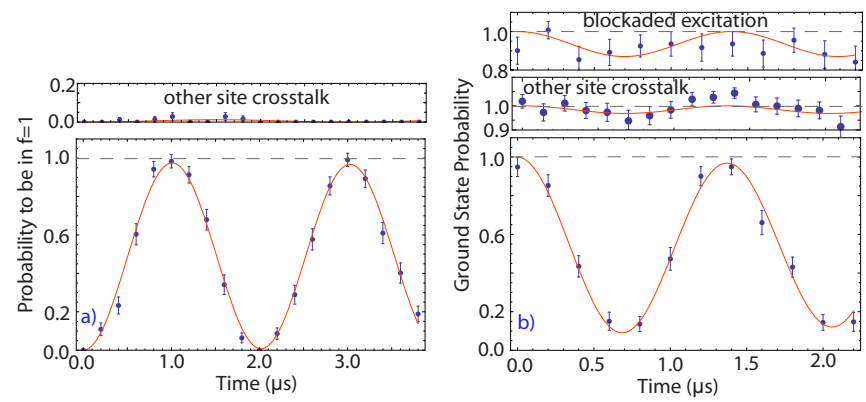

FIG. 2: (color online) a) Ground Rabi flopping on targeted site with neighboring site crosstalk in upper panel and b) Rydberg Rabi flopping on targeted site, with neighboring site crosstalk and flopping blocked by prior excitation of the neighboring site in upper panels. The flopping curves are based on an average of about 100 measurements for each point.

The CNOT table was obtained using ground state $\pi / 2$ pulses that were $\pi$ out of phase which puts the large off-diagonal values in the upper left quadrant. Correcting for background atom loss the fidelity is $F=$ $\frac{1}{4} \operatorname{Tr}\left[\left|U_{\text {ideal }}^{T}\right| U_{\mathrm{CNOT}}\right]=0.91 \pm .06$. As can be seen from Table \ the background and trace loss corrected CNOT fidelity of 0.92 agrees to within a few percent with the expected error of $1-0.06=0.94$. The error budget for the CNOT gate stems from several sources with the dominant errors due to spontaneous emission and Doppler broadening. Both of these errors could be reduced with a higher power $480 \mathrm{~nm}$ laser which would allow for faster Rydberg excitation together with larger detuning from the intermediate $5 p_{3 / 2}$ level. Since $\Omega \sim P_{\mathrm{se}} I_{480}$ where $P_{\text {se }}$ is the probability of spontaneous emission during excitation and $I_{480}$ is the intensity of the $480 \mathrm{~nm}$ laser 12 . simultaneously decreasing the spontaneous emission and the Doppler broadening errors each by a factor of ten could be achieved with an increase of about 30 times in laser intensity.

To create entangled states we used $\pi / 2$ pulses on the control atom to prepare the input states $|c t\rangle=$ $\frac{1}{\sqrt{2}}(|0\rangle+i|1\rangle)|1\rangle$ and $|c t\rangle=\frac{1}{\sqrt{2}}(|0\rangle+i|1\rangle)|0\rangle$. Applying the CNOT to these states creates two of the Bell states $\left|B_{1}\right\rangle=\frac{1}{\sqrt{2}}(|00\rangle+|11\rangle)$ and $\left|B_{2}\right\rangle=\frac{1}{\sqrt{2}}(|01\rangle+|10\rangle)$. The measured probabilities for these output states are shown in Fig. 4. In order to verify entanglement of $\left|B_{1}\right\rangle$ we measured the parity signal [19] $P=P_{00}+P_{11}-P_{01}-P_{10}$ after applying $\pi / 2$ analysis pulses to both atoms between the last CNOT pulse and the state selection pulse. The analysis pulses had a variable phase $\phi=\Omega_{A C}$, where $\Omega_{A C}=2 \pi \times 0.125 \mathrm{MHz}$ is due to AC Stark shifts from the ground state Raman beams, and $t$ is the analysis pulse gap in Fig. 4 .

A short calculation shows that the parity signal should vary as $P=2 \operatorname{Re}\left(C_{2}\right)-2\left|C_{1}\right| \cos (2 \phi+\xi)$ where $C_{2}$ is the coherence between states $|01\rangle$ and $|10\rangle$, and $C_{1}=\left|C_{1}\right| e^{\imath \xi}$ is the coherence between states $|00\rangle$ and $|11\rangle$. The parameters extracted from a least squares curve fit are given

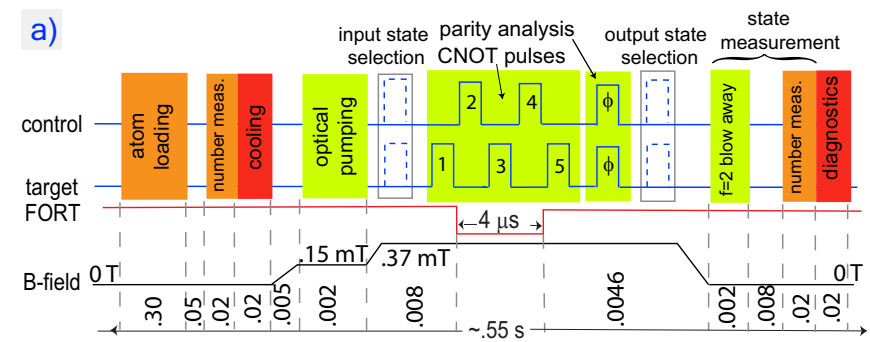

b)

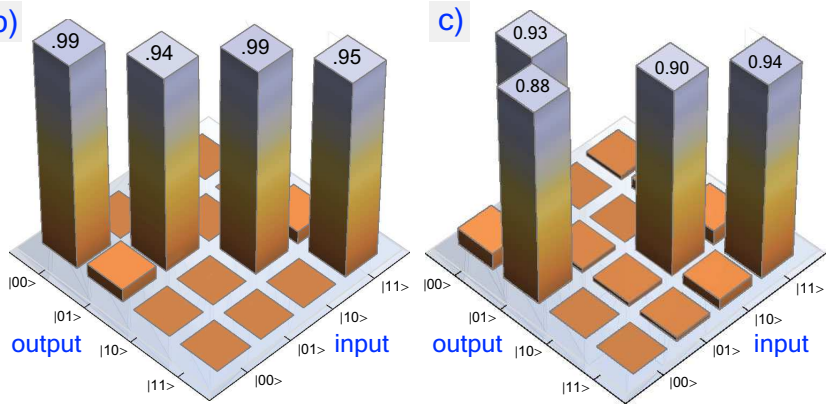

FIG. 3: (color online) a) Experimental sequence, pulses 1 and 5 are ground Rabi $\pi / 2$ pulses; pulses 2 and 4 are Rydberg $\pi$ pulses; pulse 3 is a Rydberg $2 \pi$ pulse. Measured probabilities for state preparation b) and $H-C_{Z}$ CNOT c). The reported matrices are based on an average of about 100 data points for each matrix element.

TABLE I: Error sources for the CNOT truth table and entanglement results. Spontaneous emission refers to scattering from $5 p_{3 / 2}$ during Rydberg excitation and Doppler broadening causes imperfect Rydberg excitation.

\begin{tabular}{lcc}
\hline error sources (two qubits) & Ref. [10] & this work \\
\hline \hline optical pumping & 0.1 & 0.02 \\
\hline atom losses before CNOT pulses & 0.09 & 0.02 \\
\hline blockade error at $175 \mu \mathrm{K}$ & 0.01 & 0.01 \\
\hline spontaneous emission & 0.04 & 0.04 \\
\hline Doppler broadening & 0.04 & 0.04 \\
\hline Total CNOT error (added in quadrature) & $\sim 0.15$ & $\sim 0.06$ \\
\hline measured results & & \\
\hline \hline background loss (two atoms) & 0.28 & 0.19 \\
\hline gate trace loss $(1-$ Tr $[\rho]$ ) & 0.17 & $\sim 0.01$ \\
\hline \hline CNOT fidelity raw & 0.52 & 0.74 \\
\hline CNOT background loss corrected & 0.72 & 0.91 \\
\hline CNOT background \& trace corrected & 0.86 & 0.92 \\
\hline \hline entanglement fidelity raw & 0.34 & 0.58 \\
\hline entanglement background loss corrected & 0.48 & 0.71 \\
\hline entanglement background \& trace corrected & 0.58 & 0.71 \\
\hline
\end{tabular}

in Fig. 4. The parity signal oscillates at a frequency $2 \Omega_{A C}=2 \pi \times 0.26 \mathrm{MHz}$ which is within $5 \%$ of the expected value. The phase $\xi$ of $C_{1}$ can be explained by the differential AC Stark shift induced during Rydberg excitation. Accounting for all three Rydberg pulses gives a differential phase shift between $|00\rangle$ and $|11\rangle$ of $\xi=$ 


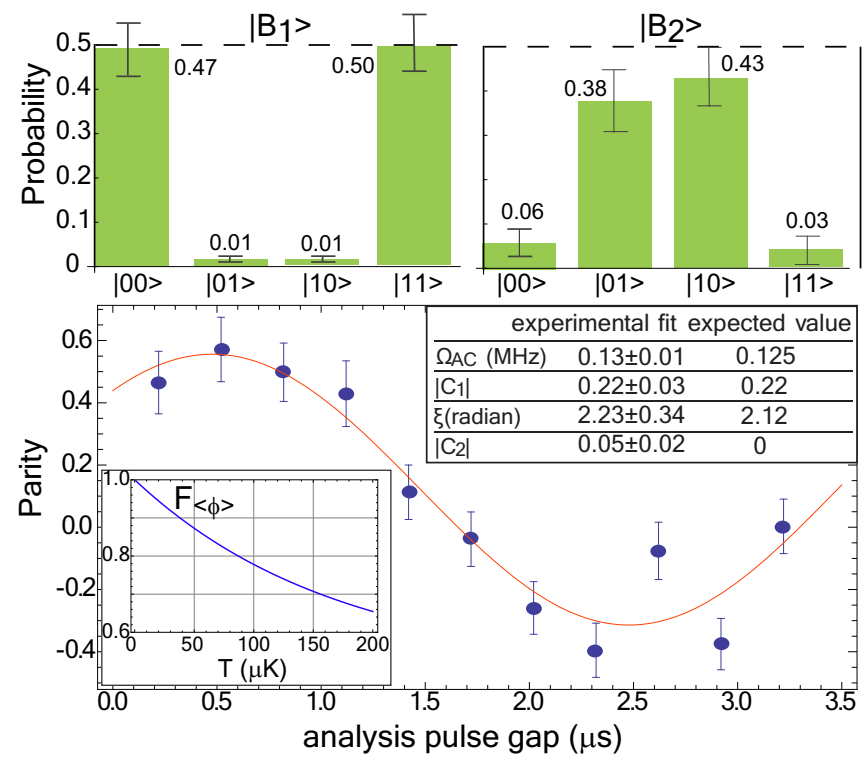

FIG. 4: (color online) Measured probabilities for preparation of Bell states $\left|B_{1}\right\rangle,\left|B_{2}\right\rangle$ and parity oscillation signal obtained from $\left|B_{1}\right\rangle$. The upper inset gives parameters found from fitting the observed parity signal together with expected values derived from measured experimental parameters. The lower inset shows the stochastic phase limited fidelity vs. temperature (see text for details).

$-2 \pi \frac{\Omega_{780}}{\Omega_{480}} \frac{\omega_{10}}{\Delta_{f=1}}=2.12+4 \pi$ which agrees with the experimental result to within $10 \%$. Here $\Omega_{780}=2 \pi \times 118 \mathrm{MHz}$, $\Omega_{480}=2 \pi \times 39 \mathrm{MHz}$ are calculated one-photon Rydberg Rabi frequencies, and $\Delta_{f=1}=\Delta_{f=2}-\omega_{10}$.

The fidelity of entanglement of the Bell state $\left|B_{1}\right\rangle$ can be quantified by $F=\frac{1}{2}\left(P_{00}+P_{11}\right)+\left|C_{1}\right|[1]$. A sufficient, but not necessary, condition for entanglement is $0.5<F \leq 1$. The population data in Fig. 4 together with the measured coherence yield $F=0.58 \pm 0.04$ without applying any corrections for atom loss or trace loss. This is the first demonstration of deterministic entanglement using Rydberg blockade. The corrected fidelities shown in Table 1 reach $F=0.71 \pm .05$ which is above the entanglement threshold by more than $4 \sigma$. The entanglement fidelity of $\left|B_{2}\right\rangle$ was not measured, but we would expect it to be somewhat lower based on the population data in Fig. 4 .

It is apparent that the fidelity of the entangled state is lower than that of the CNOT truth table, and lower than that expected from the error budget in Table I. This is due to the fact that the entanglement fidelity is sensitive to errors that do not affect the CNOT probabilities. As was pointed out in [9] the motion of Rydberg excited atoms between excitation and deexcitation pulses leads to a stochastic phase $\varphi$ that degrades the entanglement fidelity. In our implementation $\varphi=(\mathbf{k} \cdot \mathbf{v}) t_{24}$ where $|\mathbf{k}|=$ $2 \pi / \lambda_{480}-2 \pi / \lambda_{780}, \mathbf{v}$ is the atomic velocity, and $t_{24}=$
$2.2 \mu \mathrm{s}$ is the gap time between pulses 2 and 4 in Fig. 3. Assuming a temperature of $150 \mu \mathrm{K}$, which is close to the lower end of our experimental range $(140 \mu \mathrm{K}<$ $T<210 \mu \mathrm{K})$, and averaging over the thermal velocity distribution gives $\left\langle e^{\imath \varphi}\right\rangle=0.41$ which implies a maximum fidelity of $F_{<\varphi\rangle}=0.71$. Adding to this the total CNOT error from Table \ gives $F_{\max } \sim 0.65$ which is somewhat lower than our experimental result. As is seen in the inset of Fig. 4 the fidelity limit set by $F_{<\varphi>}$ depends strongly on temperature. Calculations show that reducing the atom temperature to $50 \mu \mathrm{K}$ and the gap time to $t_{24}=$ $1.5 \mu$ s would result in an entanglement fidelity above 0.9 .

In conclusion we have deterministically generated entangled states of two neutral atoms using a Rydberg blockade mediated CNOT gate. Entanglement fidelity of $F=0.58$ is obtained without any corrections for atom loss, and $F=0.71$ after accounting for losses due to collisions with background atoms. The observed fidelities are shown to be in reasonable agreement with an error model that takes into account experimental imperfections as well as the finite temperature of the atoms. Our results suggest that it should be possible to reach fidelities above 0.9 by increasing the power of the Rydberg excitation laser and better cooling of the atoms.

This work was supported by NSF grant PHY-0653408 and ARO/IARPA under contract W911NF-05-1-0492.

[1] C. A. Sackett, et al., Nature (London) 404, 256 (2000).

[2] R. Blatt and D. Wineland, Nature (London) 453, 1008 (2008).

[3] M. Steffen, et al., Science 313, 1423 (2006).

[4] P. G. Kwiat, et al., Phys. Rev. Lett. 75, 4337 (1995).

[5] B. Julsgaard, A. Kozhekin, and E. S. Polzik, Nature (London) 413, 400 (2001).

[6] O. Mandel, et al., Nature (London) 425, 937 (2003).

[7] M. Anderlini, et al., Nature (London) 448, 452 (2007).

[8] D. Jaksch, et al., Phys. Rev. Lett. 85, 2208 (2000).

[9] T. Wilk, et al., Phys. Rev. Lett. 104, 010502 (2010).

[10] L. Isenhower, et al., Phys. Rev. Lett. 104, 010503 (2010).

[11] M. Saffman and T. G. Walker, Phys. Rev. A 72, 022347 (2005).

[12] M. Saffman, T. G. Walker, and K. Mølmer, arXiv:0909.4777 (2009).

[13] T. G. Walker and M. Saffman, Phys. Rev. A 77, 032723 (2008).

[14] G. Reymond, N. Schlosser, I. Protsenko, and P. Grangier, Phil. Trans. R. Soc. Lond. A 361, 1527 (2003).

[15] E. Urban, et al., Nature Phys. 5, 110 (2009).

[16] C. Knoernschild, et al., arXiv:1006.2757 (2010).

[17] D. D. Yavuz, et al., Phys. Rev. Lett. 96, 063001 (2006).

[18] A. T. Gill, unpublished (2010).

[19] Q. A. Turchette, et al., Phys. Rev. Lett. 81, 3631 (1998). 